\title{
$N$-羟乙基- $N$-芳基丙炔酰胺的芳基磺酰化及螺-三环化反应
}

\author{
任尚峰 $a$ 王玉超 ${ }^{a}$ 刘晋彪*,a 邱观音生*,a,b \\ ( ( 江西理工大学材料冶金化学学部 江西赣州 341000) \\ ( $b$ 嘉兴学院生物与化学工程学院 浙江嘉兴 314001)
}

\begin{abstract}
摘要 报道了一种在四丁基碘化铵(TBAI)和过氧硫酸氢钾复合盐(oxone)存在下, 磺酰自由基诱导 $N$-羟乙基- $N$-芳基丙 炔酰胺的螺-三环化串联反应, 并合成了系列 1-芳基-2-对甲苯磺酰基-5,6-二氢苯并 $[b]$ 吡咯并 $[2,1-c][1,4]$ 腎嗪-3(7a $H$ )-酮. 螺-三环化串联包括磺酰自由基对炔烃的 $\alpha$-加成、ipso-环化和螺环中间体的邻位俘获.

关键词＼cjkstart螺-三环化反应; 芳基磺酰化; 自由基; 邻位捕获
\end{abstract}

\section{Arylsulfonylative spiro-Tricyclization of $\mathrm{N}$-Hydroxylethyl- $N$-arylpropiolamides}

\author{
Ren, Shangfeng ${ }^{a} \quad$ Wang, Yuchao $^{a} \quad$ Liu, Jinbiao*,a $\quad$ Qiu, Guanyinsheng ${ }^{*, a, b}$ \\ ( ${ }^{a}$ Faculty of Materials Metallurgy and Chemistry, Jiangxi University of Science and Technology, Ganzhou, Jiangxi 341000) \\ ( ${ }^{b}$ College of Biological, Chemical Sciences and Engineering, Jiaxing University, Jiaxing, Zhejiang 314001)
}

\begin{abstract}
A facile procedure is reported for the synthesis of various 1-aryl-2-tosyl-5,6-dihydrobenzo[b]pyrrolo[2,1-c][1,4]oxazin-3(7a $H)$-one via a radical arylsulfonylation-induced ipso-cyclization-ortho-cyclization sequence of $N$-hydroxylethyl- $N$ arylpropiolamides in the presence of tetra- $n$-butylammonium iodide (TBAI) and oxone. The radical cyclization sequence involves a sulfonyl radical $\alpha$-addition into the alkyne, ipso-cyclization, and ortho-trapping of the spirocyclic intermediate.

Keywords spiro-tricyclization; arylsulfonylation; radical; ortho-capture
\end{abstract}

环季碳原子广泛存在于许多有用的药物和天然产 物中 ${ }^{[1]}$. 例如 phomoidrides $\mathrm{A}$ 和 $\mathrm{B}^{[2]}$, 以及刺桐生物碱 cocculine 和 erysotrine $\mathrm{e}^{[3]}$ 都是具有广泛生物学特性的螺 旋多环化合物. 为了实现此类螺-多环化合物的合成, 化学家们付出了巨大的努力 ${ }^{[4]}$. 其中, 芳烃去芳构化是 产生螺环和季碳中心最有效的手段之一 ${ }^{[5]}$. 除了过渡金 属催化可用于芳烃的去芳构化 ${ }^{[6]}$, Larock 课题组 ${ }^{[7]}$ 较早 报道了无金属的ipso-环化(Scheme 1a), 其中亲电试剂 可以是溴和碘. 机理研究表明, 上述无金属 ispo-环化是 一种 5-endo-dig 亲电环化, 是由 4-甲氧基芳烃异构化引 发的. 这说明芳环上甲氧基在该类ipso-环化中起到了 至关重要的作用, 但也在一定程度上限制了ipso-环化 反应的应用范围. 为了实现 “普通”芳烃的 ipso-环化, 利 用过氧硫酸氢钾复合盐(oxone)化学 ${ }^{[8]}, N$-苯基丙炔酰胺
可发生溴化 ipso-环化, 顺利地生成螺-三烯酮(Scheme $1 \mathrm{~b})^{[9]}$. 尽管在上述 ipso-环化反应领域取得了较大进

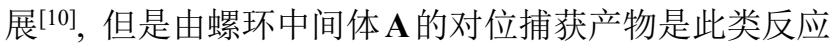
的优势物种，而邻位捕获产物较少被研究. 近期，本课 题组 ${ }^{[11]}$ 在底物设计上入手, 即在 $\mathrm{N}$ 原子上引入亲核性羟 乙基, 顺利实现了螺环中间体 $\mathbf{A}$ 的分子内邻位捕获，合 成了系列溴代螺-三环化合物(Scheme 1c).

砜作为一种极具价值的官能团，在有机化学特别是 药物化学中有着广泛的应用. 砜基团因其多样的合成应 用和重要的生物学特性而受到化学家们的广泛关注 ${ }^{[12]}$. 2015 年, 王桦课题组 ${ }^{[13]}$ 利用磺酰肼与 $N$-芳基丙炔酰胺 反应，合成了系列磺酰基取代螺环三烯酮. 本课题组 ${ }^{[14]}$ 前期主要研究了邻位酰胺(酯基)参与的芳香炔烃的多官 能团化反应，并构建了一系列有用的杂环化合物. 在近

\footnotetext{
* Corresponding authors. E-mail: liujinbiao@jxust.edu.cn; qiuguanyinsheng@mail.zjxu.edu.cn Received April 21, 2021; revised May 20, 2021; published online June 21, 2021.

Project supported by the National Natural Science Foundation of China (Nos. 21772067, 21762018, 21961014), the Natural Science Foundation of Jiangxi Province (Nos. 20171BAB213008, 20192BCBL23009, 20202BABL203005), and the Youth Jinggang Scholars Program in Jiangxi Province.

国家自然科学基金(Nos. 21772067, 21762018, 21961014)、江西省自然科学基金(Nos. 20171BAB213008, 20192BCBL23009, 20202BABL203005)、江西 省青年井冈学者奖励计划资助项目.
} 
期开发的溴代螺-三环化合物合成方法基础上 ${ }^{[11]}$, 本文 旨在报道一种磺酰自由基诱导 $N$-羟乙基- $N$-芳基丙炔酰 胺的螺-三环化串联反应, 使 $\mathbf{A}$ 中间体以邻位捕捉的方 式, 生成一系列的含砜基的螺-三环化合物的新方法 (Scheme 1d).

The previuos works:

(a)

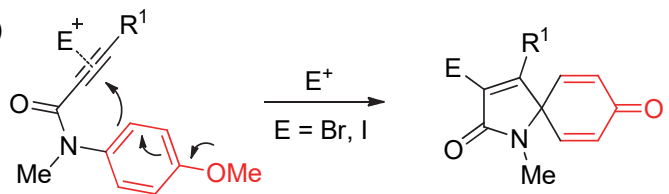

(b)

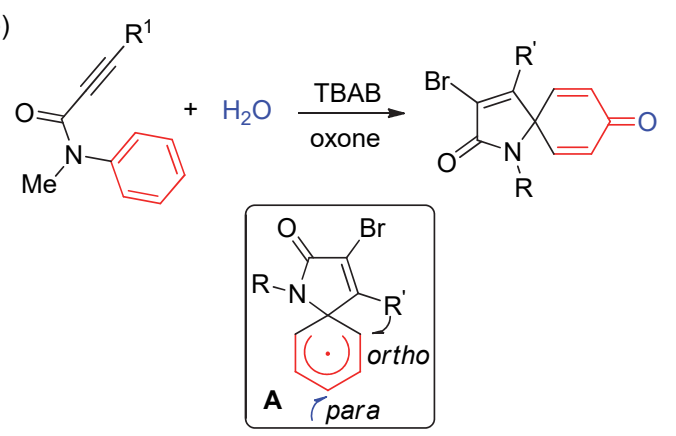

(c)

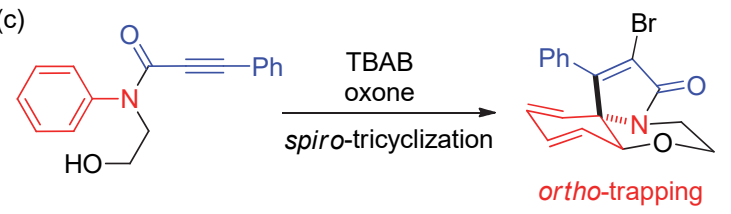

(d) This work:

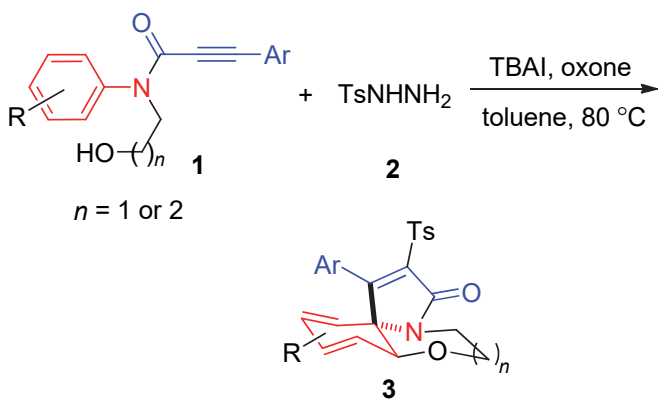

图式 $1 N$,3-二苯基丙炔酰胺的 ipso-环化反应

Scheme 1 ipso-Cyclization of N,3-diphenylpropiolamide

\section{1 结果与讨论}

首先选取化合物 1a 作为模板底物, 对甲苯磺酰胺 肼为砜基来源进行初步试验. 根据文献[13], $\mathrm{I}_{2} \mathrm{O}_{5}$ 可促进 $\mathrm{TsNHNH}_{2}$ 产生磺酰基自由基. 因此, 选择 $\mathrm{I}_{2} \mathrm{O}_{5}$ 为添加 剂、oxone 为氧化剂、1,4-二氧六环为溶剂和 $80{ }^{\circ} \mathrm{C}$ 作为 初试反应条件, 发现以 $26 \%$ 的产率得到螺-三环产物 3a (Table 1, Entry 1). 随后, 笁选了几类氧化剂, 例如 $\left(\mathrm{NH}_{4}\right)_{2} \mathrm{~S}_{2} \mathrm{O}_{8}$ 、间氯过氧苯甲酸、过氧化二苯甲酰及过氧 化二叔丁基, 但产率都未获得提高(Table 1, Entries 2
5). 紧接着对添加剂进行了考察, 发现四丁基溴化铵 (TBAB) 和单质碘作为添加剂时，反应均无法得到预期 产物 3a (Table 1, Entries 6,8). 而以四丁基碘化铵 (TBAI)作为添加剂时，产率提高到 50\% (Table 1, Entry 7). 溶剂类型对反应的影响较大, 分别考察了 1,2-二氯 乙烷、 $N, N$-二甲基甲酰胺(DMF)、甲苯和乙腈作为溶剂. 发现 DMF 作为溶剂时反应无法发生(Table 1, Entry 10); 甲苯作为溶剂时产率最高为 $53 \%$ (Table 1, Entry 11). 当 降低 TBAI 的用量到 $50 \mathrm{~mol} \%$ 时, 产物 3a 的收率降低明 显(Table 1, Entry 13). 此外, 还尝试改变反应温度, 均 未获得更好的结果(Table 1, Entries 14 16). 没有 oxone 作为氧化剂时反应无法进行(Table 1, Entry 17). 最后, 分别考察了降低氧化剂的物质的量比、氮气保护以及添 加金属路易斯酸的反应情况，但是均未得到更好的结 果. 综合考虑上述反应结果，确定反应的最佳条件：以 四丁基碘化铵为添加剂、oxone 为氧化剂、甲苯作溶剂, 在 $80{ }^{\circ} \mathrm{C}$ 下反应 $6 \mathrm{~h}$.

在最佳反应条件下，探讨了 $N$-羟乙基- $N, 3$-二芳基 丙炔酰胺 1 的螺-三环化反应范围(Table 2). 首先考察了 $N$-苯环上取代基的耐受性. 当对位为给电子甲基或乙基 时，反应以中等收率得到 $\mathbf{3 b}$ 和 $\mathbf{3} \mathbf{c}$; 当 3,5 位二甲基取代 时，产物 3d 产率可达 51\%. 当间位甲基取代时，反应得 到混合物 $\left[n(\mathbf{3 e}) / n\left(\mathbf{3} \mathbf{e}^{\prime}\right)=1 ： 1\right]$. 而对位为苯基或氟原子 取代时，产率相对较低(3f,20\%; 3h, 30\%). 当邻位苯基 取代时，反应未受到明显影响，对应产物 $\mathbf{3 g}$ 产率可得 $39 \%$. 将苯环替换为荎环时，反应亦可发生，得到 $22 \%$ 的 3i. 随后, 对亲核性羟基官能团进行了考察. 研究发 现，仲醇也可发生分子内环化得到产物 $\mathbf{3 j}$ (33\%). 并且, 羟丙基亦可顺利环化，得到 7 元环产物 3k (45\%). 对于 不同的芳基炔, $\mathrm{R}^{\prime}$ 为给电子的甲基和吸电子的氯、澳原 子以及酯基时, 都能得到相应产物(3I 30), 产率分别 为 $40 \% 、 26 \% 、 22 \%$ 和 $20 \%$. 产物 31 的确切结构经 X 单 晶衍射进一步确认(CCDC 2078592). 同样, 杂环噻吩的 引入也是可行的，得到相应产物 3p (47\%). 在实验过程 中, 发现产物 $3 p$ 在 $365 \mathrm{~nm}$ 照射下具有较好的苂光性质. 考虑到产物中含有噻吩杂环可与录离子络合, 因此考察 了产物 3p 在录离子荧光检测中的应用. 在 $\lambda_{\mathrm{ex}}=365 \mathrm{~nm}$ 激发下，往含 $3 \mathbf{p}(50 \mu \mathrm{mol} / \mathrm{L})$ 的二氯甲烷溶液中加入 1.0 equiv. 的 $\mathrm{Hg}^{2+}$ ，体系在 $450 \mathrm{~nm}$ 左右有约 2 倍的苂光增强 (Figure 1). 并且, 在紫外灯 (365 nm)照射下, 可肉眼识 别 $\mathrm{Hg}^{2+}$ 的存在. 说明化合物 $3 p$ 具备 $\mathrm{Hg}^{2+}$ 苂光检测的应 用前景.

为了进一步地了解该反应历程, 进行了 3 个对照试 验(Eqs. 1 3). 如 Eq. 1 所示, 当不加 $\mathrm{TsNHNH}_{2}$ 时，反应 未得到环化产物，这说明反应未经历中间体 $3 \mathrm{a}^{\prime}$. 当往 
表 1 反应条件的优化 ${ }^{a}$

Table 1 Optimization of the reaction conditions

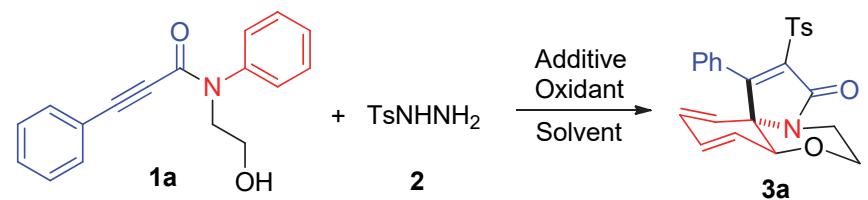

\begin{tabular}{|c|c|c|c|c|c|}
\hline Entry & Additive & Oxidant & Temp. $/{ }^{\circ} \mathrm{C}$ & Solvent & Yield $^{b}$ \\
\hline 1 & $\mathrm{I}_{2} \mathrm{O}_{5}$ & Oxone & 80 & 1,4-二氧六环 & $26 \%$ \\
\hline 2 & $\mathrm{I}_{2} \mathrm{O}_{5}$ & $\left(\mathrm{NH}_{4}\right)_{2} \mathrm{~S}_{2} \mathrm{O}_{8}$ & 80 & 1,4-二氧六环 & $12 \%$ \\
\hline 3 & $\mathrm{I}_{2} \mathrm{O}_{5}$ & 间氯过氧苯甲酸 & 80 & 1,4-二氧六环 & $15 \%$ \\
\hline 4 & $\mathrm{I}_{2} \mathrm{O}_{5}$ & 过氧化二苯甲酰 & 80 & 1,4-二氧六环 & Trace \\
\hline 5 & $\mathrm{I}_{2} \mathrm{O}_{5}$ & 过氧化二叔丁基 & 80 & 1,4-二氧六环 & $18 \%$ \\
\hline 6 & TBAB & Oxone & 80 & 1,4-二氧六环 & ND \\
\hline 7 & TBAI & Oxone & 80 & 1,4-二氧六环 & $50 \%$ \\
\hline 8 & $\mathrm{I}_{2}$ & Oxone & 80 & 1,4-二氧六环 & NR \\
\hline 9 & TBAI & Oxone & 80 & 1,2-二氯乙烷 & $36 \%$ \\
\hline 10 & TBAI & Oxone & 80 & DMF & NR \\
\hline 11 & TBAI & Oxone & 80 & 甲苯 & $53 \%$ \\
\hline 12 & TBAI & Oxone & 80 & 乙腈 & $40 \%$ \\
\hline 13 & TBAI (50 mol\%) & Oxone & 80 & 甲苯 & $26 \%$ \\
\hline 14 & TBAI & Oxone & r.t. & 甲苯 & Trace \\
\hline 15 & TBAI & Oxone & 60 & 甲苯 & $25 \%$ \\
\hline 16 & TBAI & Oxone & 100 & 甲苯 & $36 \%$ \\
\hline 17 & TBAI & - & 80 & 甲苯 & NR \\
\hline
\end{tabular}

${ }^{a}$ General conditions: 1a $(0.2 \mathrm{mmol}), \mathbf{2}(0.6 \mathrm{mmol})$, additive $(0.2 \mathrm{mmol})$, oxidant $(0.6 \mathrm{mmol})$ in solvent $(2 \mathrm{~mL})$, stirred for 6 h. ${ }^{b}$ Isolated yield.

表 2 反应底物的范围 $a$

Table 2 Reaction substrate scopes
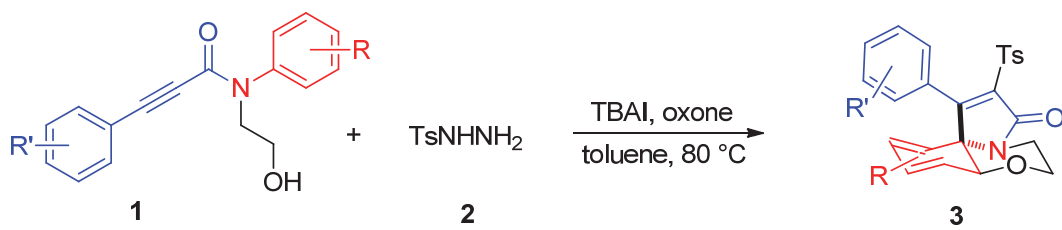

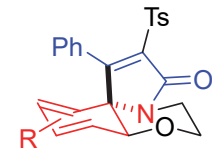

3a, $\mathrm{R}=\mathrm{H}, 53 \% ; 3 \mathrm{~b}, \mathrm{R}=4-\mathrm{Me}, 52 \%$

3c, $R=4-E t, 42 \% ; 3 f, R=4-P h, 20 \%$

3 g, R $=2-\mathrm{Ph}, 39 \% ; 3 \mathrm{~h}, \mathrm{R}=4-\mathrm{F}, 30 \%$

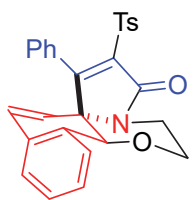

$3 i, 22 \%$

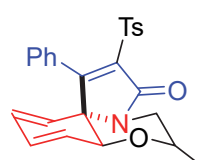

$3 \mathbf{j}, 33 \%$

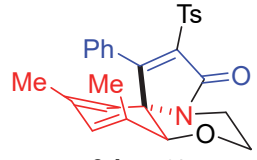

3d, $51 \%$

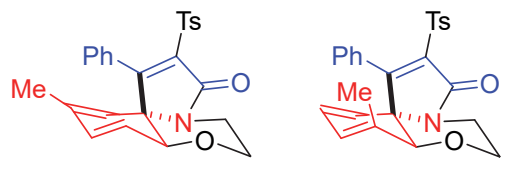

$3 \mathbf{e} / 3 \mathbf{e}^{\prime}=1 / 1,44 \%$

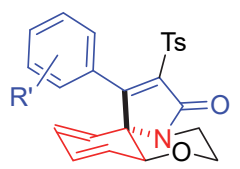

3I, $\mathrm{R}^{\prime}=4-\mathrm{Me}, 40 \%$

$3 \mathrm{~m}, \mathrm{R}^{\prime}=4-\mathrm{Cl}, 26 \%$

$3 n, R^{\prime}=4-B r, 22 \%$

3o, $\mathrm{R}^{\prime}=4-\mathrm{COOMe}, 20 \%$

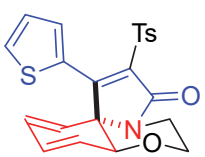

$3 p, 47 \%$

${ }^{a}$ The reaction was performed with $1(0.2 \mathrm{mmol}), 2(0.6 \mathrm{mmol})$, TBAI $(0.2 \mathrm{mmol})$, oxone $(0.6 \mathrm{mmol})$ in toluene $(2 \mathrm{~mL})$ at $80{ }^{\circ} \mathrm{C}$ for $6 \mathrm{~h} .{ }^{b}$ Isolated yield.

模板反应中添加 2.0 equiv. 2,2,6,6-四甲基哌啶氧化物 (TEMPO)时，检测到反应产率降低明显，产率仅为 $12 \%$ (Eq. 2). 这说明该反应很有可能经历了自由基过程. 当 反应以对甲苯亚磺酸钠替代 $\mathrm{TsNHNH}_{2}$ 作为砜源，反应
亦可发生, 并得到 27\%的收率(Eq. 3).

基于上述反应结果以及相关文献 ${ }^{[11-14]}$, 我们提出了 可能的反应机理(Scheme 2). 首先 $\mathrm{I}^{-}$在 oxone 氧化下生 成硒自由基，随后 $\mathrm{TsNHNH}_{2}$ 在碘自由基和 oxone 作用下 


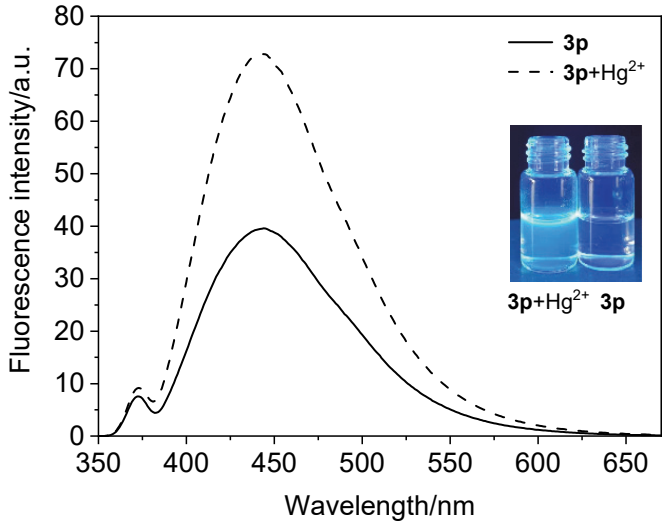

图 1 化合物 $3 p$ 用于采离子的苂光检测

Figure 1 Compound $3 p$ used for fluorescence detection of $\mathrm{Hg}^{2+}$<smiles>O=C(C#Cc1ccccc1)N(CCO)c1ccccc1</smiles>

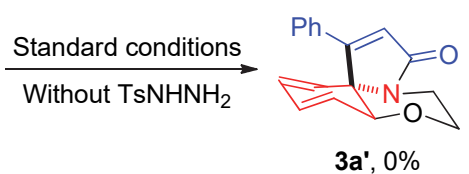

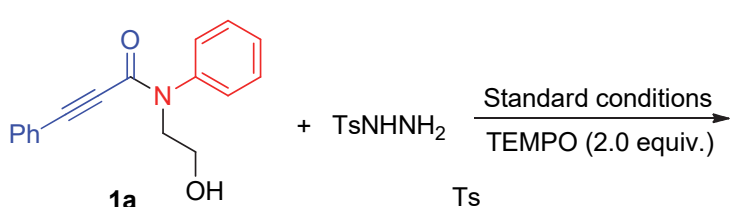

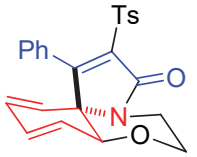

3a, $12 \%$<smiles>O=C(C#Cc1ccccc1)N(CCO)c1ccccc1</smiles><smiles>Cc1ccc(S(=O)(=O)Nc2ccccc2)cc1</smiles>

Standard conditions

$1 \mathrm{a}$<smiles>[3H]c1c(-c2ccccc2)c2ccccc2n(CCO)c1=O</smiles>

$3 a, 27 \%$

发生氧化和脱氮, 得到砜自由基. 砜自由基再对 1a 炔键 进行自由基加成得到中间体 $\mathbf{I}$, 紧接着发生芳胺的 ipso环化得到芳基自由基中间体 II; oxone 再将自由基中间 体氧化成正离子中间体 III. 最终羟基邻位进攻苯环, 并 脱去质子得到目标产物 3 a.

为了进一步验证该反应的应用性, 进行了放大实 验: 在同样的条件下, 把原料投料量放大五倍, 产率同 样可以达到 50\%, 并得到产物 3a $210 \mathrm{mg}$ (Eq. 4).

\section{2 结论}

在 TBAI 和 oxone 存在下, $\mathrm{TsNHNH}_{2}$ 与 $\mathrm{N}$-羟乙基- $\mathrm{N}$ 芳基丙炔酰胺可发生螺-三环化串联反应，合成系列 1-

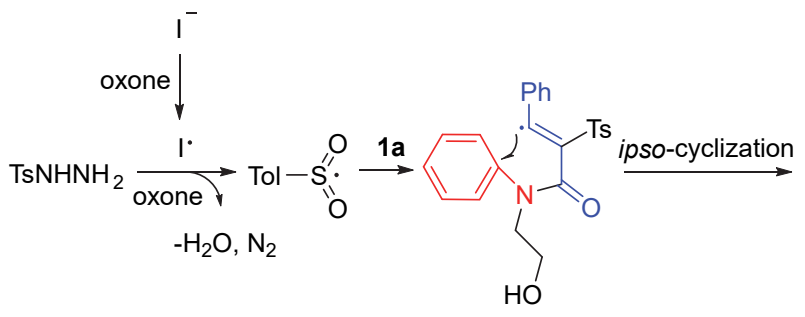

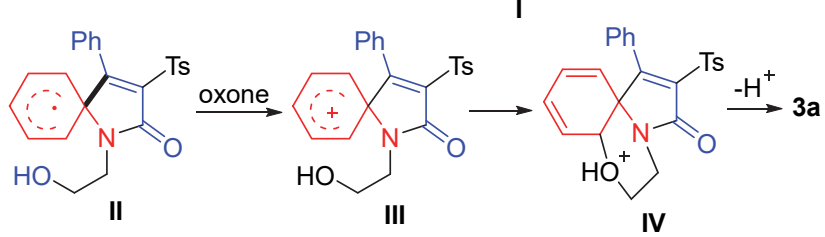

图式 2 可能的反应机理 Scheme 2 Proposed reaction mechanism

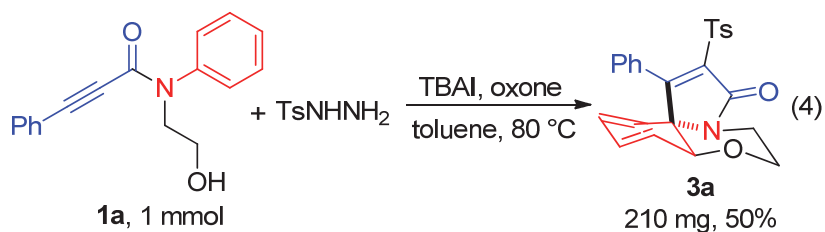

芳基-2-对甲苯磺酰基-5,6-二氢苯并 $[b]$ 吡咯并 $[2,1$ $c][1,4]$ 惡嗪-3(7a $H)$-酮. 该螺-三环化串联过程包括磺酰 自由基对炔烃的 $\alpha$-加成、ipso-环化和螺环中间体的邻位 俘获. 此类螺-三环骨架化合物的生物学应用还在进一 步的实验当中.

\section{3 实验部分}

\section{1 仪器与试剂}

除非另有说明，所用试剂均为市售分析纯，未进一 步纯化. 在硅胶 (200 300 目)上通过快速色谱法对产物 进行纯化. 在 Bruker Avance III 400(400 MHz)上测量了 核磁共振(NMR)谱. ${ }^{1} \mathrm{H}$ NMR (400 MHz) 和 ${ }^{13} \mathrm{C}$ NMR $(100$ $\mathrm{MHz}$ ) 所用溶剂为 $\mathrm{CDCl}_{3}, \mathrm{TMS}$ 为内标. HRMS 数据在 VG ZAB-HS 质谱仪、Brucker Apex IV FTMS 光谱仪上 获得. $\mathrm{X}$ 射线单晶衍射数据收集在安捷伦科技公司 Gemini 单晶衍射仪上获得. 荧光光谱在 F-4600 荧光分 光光度计上测得.

\section{2 实验方法}

向烧瓶中加入 $1(0.2 \mathrm{mmol})$ 、磺酰肼 $(0.6 \mathrm{mmol})$ 、 oxone $(0.6 \mathrm{mmol}) 、 \mathrm{TBAI}(0.2 \mathrm{mmol})$ 和甲苯 $(2 \mathrm{~mL})$, 然后 将反应混合物加热至 $80{ }^{\circ} \mathrm{C}$, 搅拌 $6 \mathrm{~h}$. 冷却至室温后, 用水 $(5 \mathrm{~mL})$ 和乙酸乙酯 $(5 \mathrm{~mL} \times 3)$ 处理混合物. 有机提 取物在 $\mathrm{Na}_{2} \mathrm{SO}_{4}$ 上干燥并旋蒸浓缩, 最后经柱层析得到 目标产物 3.

1-苯基-2-对甲苯磺酰基-5,6-二氢苯并 $[b]$ 吡咯并 
[2,1-c][1,4]悪嗪-3(7aH)-酮(3a): 白色固体, $42.6 \mathrm{mg}$, 产 率 53\%. m.p. 217 $219{ }^{\circ} \mathrm{C} ;{ }^{1} \mathrm{H}$ NMR (400 MHz, $\mathrm{CDCl}_{3}$ ) $\delta: 7.87(\mathrm{~d}, J=7.7 \mathrm{~Hz}, 2 \mathrm{H}), 7.39$ (d, $J=7.2 \mathrm{~Hz}, 1 \mathrm{H}), 7.30$ $(\mathrm{d}, J=6.4 \mathrm{~Hz}, 4 \mathrm{H}), 7.08(\mathrm{~d}, J=7.2 \mathrm{~Hz}, 2 \mathrm{H}), 6.25$ (d, $J=$ $8.4 \mathrm{~Hz}, 1 \mathrm{H}), 5.60$ (s, 2H), 5.35 (d, $J=9.2 \mathrm{~Hz}, 1 \mathrm{H}), 4.24 \sim$ $4.21(\mathrm{~m}, 1 \mathrm{H}), 3.86(\mathrm{~d}, J=6.8 \mathrm{~Hz}, 1 \mathrm{H}), 3.73(\mathrm{~d}, J=2.6 \mathrm{~Hz}$, $1 \mathrm{H}), 3.35 \sim 3.32(\mathrm{~m}, 1 \mathrm{H}), 3.15 \sim 3.09(\mathrm{~m}, 1 \mathrm{H}), 2.42(\mathrm{~s}$, $3 \mathrm{H}) ;{ }^{13} \mathrm{C}$ NMR (101 MHz, $\left.\mathrm{CDCl}_{3}\right) \delta: 163.6,162.3,145.2$, $136.8,135.2$, 130.5, 129.9, 129.6, 129.1, 129.0, 127.6, $127.0,126.5,124.7,123.01,72.8,67.4,66.3,39.2$, 21.8; IR (KBr) v: 3054, 2959, 2920, 2851, 1703, 1620, 1593, 1491, 1442, 1390, 1325, 1296, 1159, 1148, 1063, 962, 816, 767, $720,703 \mathrm{~cm}^{-1}$; HRMS (ESI-TOF) calcd for $\mathrm{C}_{24} \mathrm{H}_{21} \mathrm{NO}_{4-}$ $\mathrm{NaS}[\mathrm{M}+\mathrm{Na}]^{+}$442.1086, found 442.1089.

9-甲基-1-苯基-2-对甲苯磺酰基-5,6-二氢苯并 $[b]$ 吡 咯并[2,1-c][1,4]檼嗪-3(7aH)-酮 $(3 \mathbf{b})$ : 白色固体, $45.1 \mathrm{mg}$, 产率 52\%. m.p. $237 \sim 239{ }^{\circ} \mathrm{C} ;{ }^{1} \mathrm{H}$ NMR $(400 \mathrm{MHz}$, $\left.\mathrm{CDCl}_{3}\right) \delta: 7.86(\mathrm{~d}, J=8.0 \mathrm{~Hz}, 2 \mathrm{H}), 7.36 \sim 7.34(\mathrm{~m}, 1 \mathrm{H})$, $7.30 \sim 7.28(\mathrm{~m}, 4 \mathrm{H}), 7.07(\mathrm{~d}, J=7.2 \mathrm{~Hz}, 2 \mathrm{H}), 6.11(\mathrm{~d}, J=$ $9.5 \mathrm{~Hz}, 1 \mathrm{H}), 5.35$ (d, $J=9.4 \mathrm{~Hz}, 1 \mathrm{H}), 5.29$ (d, $J=4.7 \mathrm{~Hz}$, $1 \mathrm{H}), 4.22 \sim 4.19(\mathrm{~m}, 1 \mathrm{H}), 3.84 \sim 3.82(\mathrm{~m}, 1 \mathrm{H}), 3.63(\mathrm{~s}$, $1 \mathrm{H}), 3.33 \sim 3.30(\mathrm{~m}, 1 \mathrm{H}), 3.12 \sim 3.07(\mathrm{~m}, 1 \mathrm{H}), 2.41(\mathrm{~s}$, $3 \mathrm{H}), 1.30(\mathrm{~s}, 3 \mathrm{H}) ;{ }^{13} \mathrm{C} \mathrm{NMR}\left(101 \mathrm{MHz}, \mathrm{CDCl}_{3}\right) \delta: 163.7$, $162.3,145.1,136.7,136.4,135.1,134.6,130.0,129.6$, $129.0,128.9,127.6,126.9,122.6,119.2,73.8,67.5,66.2$, $39.2,21.75,20.7$; IR (KBr) v: 3047, 3032, 2971, 2919, 2862, 1694, 1622, 1593, 1492, 1443, 1391, 1327, 1290, 1196, 1178, 1160, 1148, 1089, 1056, 958, 820, 768, 743; HRMS (ESI-TOF) calcd for $\mathrm{C}_{25} \mathrm{H}_{23} \mathrm{NO}_{4} \mathrm{NaS}[\mathrm{M}+\mathrm{Na}]^{+}$ 456.1246, found 456.1245.

9-乙基-1-苯基-2-对甲苯磺酰基-5,6-二氢苯并 $[b]$ 吡 咯并 $[2,1-c][1,4]$ 噁嗪-3(7aH)-酮(3c): 白色固体, $37.6 \mathrm{mg}$, 产率 42\%. m.p. 243 $245{ }^{\circ} \mathrm{C}$; ${ }^{1} \mathrm{H}$ NMR $(400 \mathrm{MHz}$, $\left.\mathrm{CDCl}_{3}\right) \delta: 7.86(\mathrm{~d}, J=7.2 \mathrm{~Hz}, 2 \mathrm{H}), 7.35(\mathrm{~d}, J=6.9 \mathrm{~Hz}$, 1H), 7.30 (d, $J=6.7 \mathrm{~Hz}, 4 \mathrm{H}), 7.06$ (d, $J=6.7 \mathrm{~Hz}, 2 \mathrm{H}), 6.13$ $(\mathrm{d}, J=9.3 \mathrm{~Hz}, 1 \mathrm{H}), 5.36(\mathrm{~d}, J=9.7 \mathrm{~Hz}, 1 \mathrm{H}), 5.32$ (d, $J=$ $3.6 \mathrm{~Hz}, 1 \mathrm{H}), 4.22$ (d, $J=13.1 \mathrm{~Hz}, 1 \mathrm{H}), 3.84$ (d, $J=10.5$ $\mathrm{Hz}, 1 \mathrm{H}), 3.70(\mathrm{~d}, J=4.6 \mathrm{~Hz}, 1 \mathrm{H}), 3.34 \sim 3.28(\mathrm{~m}, 1 \mathrm{H})$, $3.13 \sim 3.07(\mathrm{~m}, 1 \mathrm{H}), 2.42(\mathrm{~s}, 3 \mathrm{H}), 1.73 \sim 1.59(\mathrm{~m}, 2 \mathrm{H})$, $0.51(\mathrm{t}, J=6.8 \mathrm{~Hz}, 3 \mathrm{H}) ;{ }^{13} \mathrm{C} \mathrm{NMR}\left(101 \mathrm{MHz}, \mathrm{CDCl}_{3}\right) \delta$ : $163.9,162.3,145.1,141.7,136.7,135.2,133.8,130.0$, 129.6, 129.0, 128.9, 127.6, 126.9, 122.7, 117.7, 73.7, 67.7, 66.2, 39.2, 27.7, 21.8, 11.3; IR (KBr) v: 3047, 3032, 2971, 2919, 2862, 1692, 1622, 1593, 1492, 1443, 1391, 1327, $120,1196,1178,1160,1148,1089,1056,958,820$; HRMS
(ESI-TOF) calcd for $\mathrm{C}_{26} \mathrm{H}_{25} \mathrm{NO}_{4} \mathrm{NaS}[\mathrm{M}+\mathrm{Na}]^{+}$470.1397, found 470.1402 .

8,10-二甲基-1-苯基-2-对甲苯磺酰基-5,6-二氢苯并 $[b]$ 吡咯并 $[2,1-c][1,4]$ 噁嗪 $-3(7 \mathrm{a} H)$-酮 $(3 \mathbf{d})$ : 白色固体, $49.1 \mathrm{mg}$, 产率 55\%. m.p. 227 $229{ }^{\circ} \mathrm{C} ;{ }^{1} \mathrm{H}$ NMR $(400$ $\left.\mathrm{MHz}, \mathrm{CDCl}_{3}\right) \delta: 7.84(\mathrm{~d}, J=8.2 \mathrm{~Hz}, 2 \mathrm{H}), 7.34$ (d, $J=7.3$ $\mathrm{Hz}, 1 \mathrm{H}), 7.29 \sim 7.25(\mathrm{~m}, 4 \mathrm{H}), 7.00 \sim 6.96(\mathrm{~m}, 2 \mathrm{H}), 5.05(\mathrm{~s}$, $1 \mathrm{H}), 4.85(\mathrm{~s}, 1 \mathrm{H}), 4.16 \sim 4.13(\mathrm{~m}, 1 \mathrm{H}), 3.85 \sim 3.81(\mathrm{~m}$, $1 \mathrm{H}), 3.27(\mathrm{~s}, 1 \mathrm{H}), 3.21 \sim 3.27(\mathrm{~m}, 1 \mathrm{H}), 3.11 \sim 3.04(\mathrm{~m}$, 1H), $2.38(\mathrm{~s}, 3 \mathrm{H}), 1.73(\mathrm{~s}, 3 \mathrm{H}), 1.53(\mathrm{~s}, 3 \mathrm{H}) ;{ }^{13} \mathrm{C} \mathrm{NMR}$ $\left(101 \mathrm{MHz}, \mathrm{CDCl}_{3}\right) \delta: 164.5,162.1,145.0,140.1,136.8$, $134.4,134.4,130.2,129.6,129.1,128.9,126.9,126.6$, $125.9,113.4,77.43,68.7,66.4,39.1,21.8,21.2,20.5$; IR (KBr) v: 3441, 2928, 2861, 1702, 1631, 1593, 1440, 1399, 1331, 1293, 1185, 1160, 1087, 1072, 1057, 968, 769, 703, $657,587,543$; HRMS (ESI-TOF) calcd for $\mathrm{C}_{26} \mathrm{H}_{25} \mathrm{NO}_{4} \mathrm{NaS}$ $[\mathrm{M}+\mathrm{Na}]^{+}$470.1399, found 470.1402 .

8-甲基-1-苯基-2-对甲苯磺酰基-5,6-二氢苯并 $[b]$ 吡 咯并 $[2,1-c][1,4]$ 啞嗪-3(7aH)-酮(3e)和 10-甲基-1-苯基-2甲苯磺酰基-5,6-二氢苯并 $[b]$ 吡咯并 $[2,1-c][1,4]$ 啞嗪3(7aH)-酮 $\left(3 \mathrm{e}^{\prime}\right)$ : 白色固体, $47.0 \mathrm{mg}$, 产率 44\%. m.p. $178 \sim 179{ }^{\circ} \mathrm{C} ;{ }^{1} \mathrm{H}$ NMR $\left(400 \mathrm{MHz}, \mathrm{CDCl}_{3}\right) \delta: 7.85$ (d, $J=$ $8.2,1.1 \mathrm{~Hz}, 4 \mathrm{H}), 7.39 \sim 7.35(\mathrm{~m}, 2 \mathrm{H}), 7.32 \sim 7.26(\mathrm{~m}, 8 \mathrm{H})$, $7.06 \sim 7.00(\mathrm{~m}, 4 \mathrm{H}), 6.19 \sim 6.15(\mathrm{~m}, 1 \mathrm{H}), 5.57 \sim 5.53(\mathrm{~m}$, $1 \mathrm{H}), 5.40 \sim 5.38(\mathrm{~m}, 1 \mathrm{H}), 5.24(\mathrm{~d}, J=4.2 \mathrm{~Hz}, 1 \mathrm{H}), 5.18(\mathrm{~d}$, $J=9.4 \mathrm{~Hz}, 1 \mathrm{H}), 5.01(\mathrm{~s}, 1 \mathrm{H}), 4.20 \sim 4.17(\mathrm{~m}, 2 \mathrm{H}), 3.88 \sim$ $3.81(\mathrm{~m}, 2 \mathrm{H}), 3.65(\mathrm{~s}, 1 \mathrm{H}), 3.40(\mathrm{~s}, 1 \mathrm{H}), 3.32 \sim 3.25(\mathrm{~m}$, $2 \mathrm{H}), 3.13 \sim 3.06(\mathrm{~m}, 2 \mathrm{H}), 2.40(\mathrm{~s}, 6 \mathrm{H}), 1.79(\mathrm{~s}, 3 \mathrm{H}), 1.56$ $(\mathrm{s}, 3 \mathrm{H}) ;{ }^{13} \mathrm{C}$ NMR $\left(101 \mathrm{MHz}, \mathrm{CDCl}_{3}\right) \delta: 164.3,163.7$, $162.3,162.1,162.1,145.1,145.1,145.1,145.1,139.2$, $139.2,136.8,136.8,136.7,136.7,134.9,134.8,134.7$, $134.7,131.3,131.3,130.7,130.7,130.2,130.2,129.9$, $129.9,129.6,129.6,129.2,129.2,129.0,129.0,129.0$, $127.4,127.4,126.9,126.9,126.9,126.8,124.3,124.3$, $121.7,121.7,119.4,119.4,116.8,116.8,77.8,77.8,77.4$, $77.1,76.8,72.4,72.4,68.2,68.2,67.8,67.8,66.5,66.5$, $66.2,66.2,39.2,39.2,39.1,39.1,21.8,21.8,21.2,21.2$, 20.7, 20.7; IR (KBr) v: 3447, 2925, 1702, 1631, 1594, $1490,1441,1385,1329,1289,1156,1073,967,928,894$, 815, 767, 735, 703, 694, 660, 584, 541; HRMS (ESI-TOF) calcd for $\mathrm{C}_{25} \mathrm{H}_{23} \mathrm{NO}_{4} \mathrm{NaS}[\mathrm{M}+\mathrm{Na}]^{+}$456.1253, found 456.1245 .

1,9-二苯基-2-对甲苯磺酰基-5,6-二氢苯并 $[b]$ 吡咯 并 $[2,1-c][1,4]$ 啞嗪-3(7aH)-酮(3f): 白色固体, $19.8 \mathrm{mg}$, 产率 $20 \%$. m.p. $238 \sim 240{ }^{\circ} \mathrm{C} ;{ }^{1} \mathrm{H}$ NMR $(400 \mathrm{MHz}$, 
$\left.\mathrm{CDCl}_{3}\right) \delta: 7.86(\mathrm{~d}, J=7.0 \mathrm{~Hz}, 2 \mathrm{H}), 7.29(\mathrm{~d}, J=7.6 \mathrm{~Hz}$, 2H), $7.24 \sim 7.18(\mathrm{~m}, 7 \mathrm{H}), 7.10(\mathrm{~d}, J=5.0 \mathrm{~Hz}, 2 \mathrm{H}), 6.83(\mathrm{~d}$, $J=7.2 \mathrm{~Hz}, 2 \mathrm{H}), 6.58$ (d, $J=9.6 \mathrm{~Hz}, 1 \mathrm{H}), 5.69$ (d, $J=5.7$ $\mathrm{Hz}, 1 \mathrm{H}), 5.52(\mathrm{~d}, J=9.6 \mathrm{~Hz}, 1 \mathrm{H}), 4.26 \sim 4.23(\mathrm{~m}, 1 \mathrm{H})$, $3.90 \sim 3.85(\mathrm{~m}, 2 \mathrm{H}), 3.38 \sim 3.32(\mathrm{~m}, 1 \mathrm{H}), 3.19 \sim 3.13(\mathrm{~m}$, $1 \mathrm{H}), 2.41(\mathrm{~s}, 3 \mathrm{H}) ;{ }^{13} \mathrm{C} \mathrm{NMR}\left(101 \mathrm{MHz}, \mathrm{CDCl}_{3}\right) \delta: 163.6$, $162.4,145.2,139.5,138.1,136.7,135.3,132.9,129.9$, $129.6,129.1,129.0,128.2,128.1,127.4,127.2,125.9$, $123.8, \quad 120.1,73.7,67.3,66.3,39.2,21.8$; HRMS (ESI-TOF) calcd for $\mathrm{C}_{30} \mathrm{H}_{25} \mathrm{NO}_{4} \mathrm{NaS}[\mathrm{M}+\mathrm{Na}]^{+}$518.1408, found 518.1402.

1,11-二苯基-2-对甲苯磺酰基-5,6-二氢苯并 [ $b$ 吡咯 并 $[2,1-c][1,4]$ 憩嗪-3(7a $H)$-酮 $(3 \mathrm{~g})$ : 白色固体, $38.6 \mathrm{mg}$, 产率 39\%. m.p. 243 244 ${ }^{\circ} \mathrm{C}$; ${ }^{1} \mathrm{H}$ NMR $(400 \mathrm{MHz}$, $\left.\mathrm{CDCl}_{3}\right) \delta: 7.90(\mathrm{~d}, J=7.3 \mathrm{~Hz}, 2 \mathrm{H}), 7.42 \sim 7.36(\mathrm{~m}, 3 \mathrm{H})$, $7.31 \sim 7.21(\mathrm{~m}, 2 \mathrm{H}), 7.24 \sim 7.20(\mathrm{~m}, 1 \mathrm{H}), 7.11(\mathrm{~d}, J=7.1$ $\mathrm{Hz}, 2 \mathrm{H}), 7.04 \sim 7.01(\mathrm{~m}, 2 \mathrm{H}), 6.86(\mathrm{~d}, J=8.2 \mathrm{~Hz}, 2 \mathrm{H})$, $6.32(\mathrm{~d}, J=4.9 \mathrm{~Hz}, 1 \mathrm{H}), 5.68 \sim 5.63(\mathrm{~m}, 2 \mathrm{H}), 5.27(\mathrm{~s}, 1 \mathrm{H})$, $3.91 \sim 3.87(\mathrm{~m}, 1 \mathrm{H}), 3.82 \sim 3.78(\mathrm{~m}, 1 \mathrm{H}), 3.68 \sim 3.67(\mathrm{~m}$, $1 \mathrm{H}), 3.37 \sim 3.32(\mathrm{~m}, 1 \mathrm{H}), 2.48(\mathrm{~s}, 3 \mathrm{H}) ;{ }^{13} \mathrm{C}$ NMR $(101$ $\left.\mathrm{MHz}, \mathrm{CDCl}_{3}\right) \delta: 164.7,162.6,145.2,137.2,136.6,135.4$, $132.4,129.8,129.5,129.5,129.4,128.9,128.9,128.4$, 128.3, 127.7, 127.1, 126.3, 124.1, 76.0, 69.3, 66.6, 39.9, 21.8; IR (KBr) v: 3400, 3053, 2924, 2873, 1700, 1618, 1593, 1490, 1442, 1398, 1386, 1331, 1290, 1162, 1088, 1066, 975, 811, 722, 695, $663 \mathrm{~cm}^{-1}$; HRMS (ESI-TOF) calcd for $\mathrm{C}_{30} \mathrm{H}_{25} \mathrm{NO}_{4} \mathrm{NaS}[\mathrm{M}+\mathrm{Na}]^{+}$518.1407, found 518.1402.

9-氟-1-苯基-2-对甲苯磺酰基-5,6-二氢苯并 $[b]$ 吡咯 并[2,1-c][1,4]唓嗪-3(7a $H)$-酮 (3h): 白色固体, $26.2 \mathrm{mg}$, 产率 30\%. m.p. $225 \sim 227{ }^{\circ} \mathrm{C} ;{ }^{1} \mathrm{H}$ NMR $(400 \mathrm{MHz}$, $\left.\mathrm{CDCl}_{3}\right) \delta: 7.84(\mathrm{~d}, J=7.7 \mathrm{~Hz}, 2 \mathrm{H}), 7.40(\mathrm{~d}, J=7.1 \mathrm{~Hz}$, $1 \mathrm{H}), 7.35 \sim 7.28(\mathrm{~m}, 4 \mathrm{H}), 7.06(\mathrm{~d}, J=7.2 \mathrm{~Hz}, 2 \mathrm{H}), 6.20 \sim$ $6.09(\mathrm{~m}, 1 \mathrm{H}), 5.50(\mathrm{~s}, 1 \mathrm{H}), 5.12 \sim 4.98(\mathrm{~m}, 1 \mathrm{H}), 4.21(\mathrm{~d}$, $J=13.3 \mathrm{~Hz}, 1 \mathrm{H}), 3.84(\mathrm{~d}, J=10.2 \mathrm{~Hz}, 2 \mathrm{H}), 3.33 \sim 3.27(\mathrm{~m}$, $1 \mathrm{H}), 3.11 \sim 3.05(\mathrm{~m}, 1 \mathrm{H}), 2.40(\mathrm{~s}, 3 \mathrm{H}) ;{ }^{13} \mathrm{C}$ NMR $(101$ $\left.\mathrm{MHz}, \mathrm{CDCl}_{3}\right) \delta: 162.5(\mathrm{~d}, J=45.6 \mathrm{~Hz}), 160.8,158.2$, $145.3,136.5,135.8,129.7,129.4,129.3,129.0,127.5$, $127.4,127.3,127.0,100.8$ (d, $J=19.0 \mathrm{~Hz}), 74.5$ (d, $J=$ $12.7 \mathrm{~Hz}), 67.3,66.2,39.3,21.8$; IR (KBr) v: 3429, 2982, 2924, 2864, 1708, 1673, 1631, 1595, 1492, 1442, 1401, 1319, 1292, 1199, 1159, 1179, 1147, 1121, 1085, 1041, 961, 933, 901, 817, 783, 767, 748, $695 \mathrm{~cm}^{-1}$; HRMS (ESITOF) calcd for $\mathrm{C}_{24} \mathrm{H}_{20} \mathrm{NO}_{4} \mathrm{NaSF}[\mathrm{M}+\mathrm{Na}]^{+}$460.0999, found 460.0995 .
7-苯基-6-对甲苯磺酰基-2,3-二氢荎并 $[1,2-b]$ 吡咯并 [2,1-c][1,4]慧嗪-5(13bH)-酮(3i): 白色固体, $20.6 \mathrm{mg}$, 产 率 22\%. m.p. $255 \sim 256{ }^{\circ} \mathrm{C} ;{ }^{1} \mathrm{H}$ NMR $\left(400 \mathrm{MHz}, \mathrm{CDCl}_{3}\right)$ $\delta: 7.86(\mathrm{~d}, J=8.0 \mathrm{~Hz}, 2 \mathrm{H}), 7.29(\mathrm{~d}, J=8.0 \mathrm{~Hz}, 2 \mathrm{H}), 7.03 \sim$ $6.96(\mathrm{~m}, 6 \mathrm{H}), 6.87 \sim 6.85(\mathrm{~m}, 2 \mathrm{H}), 6.73 \sim 6.69(\mathrm{~m}, 2 \mathrm{H})$, $5.38(\mathrm{~d}, J=9.2 \mathrm{~Hz}, 1 \mathrm{H}), 4.26 \sim 4.23(\mathrm{~m}, 1 \mathrm{H}), 4.10(\mathrm{~s}, 1 \mathrm{H})$, $3.92 \sim 3.88(\mathrm{~m}, 1 \mathrm{H}), 3.50 \sim 3.45(\mathrm{~m}, 1 \mathrm{H}), 3.21 \sim 3.15(\mathrm{~m}$, $1 \mathrm{H}), 2.41(\mathrm{~s}, 3 \mathrm{H}) ;{ }^{13} \mathrm{C}$ NMR $\left(101 \mathrm{MHz}, \mathrm{CDCl}_{3}\right) \delta: 163.8$, $162.3,145.2,136.7,135.1,134.6,131.5,131.3,129.7$, $129.6,129.1,129.1,128.8,128.8,128.5,127.3,127.0$, 126.6, 121.7, 78.6, 68.3, 66.4, 38.8, 21.8; IR (KBr) v: $3435,3051,2977,2928,2860,1695,1620,1596,1490$, $1455,1440,1397,1328,1287,1186,1161,1147,1089$, 1061, 969, 952, 899, 809, 778, 766, $731 \mathrm{~cm}^{-1}$; HRMS (ESI-TOF) calcd for $\mathrm{C}_{28} \mathrm{H}_{23} \mathrm{NO}_{4} \mathrm{NaS}[\mathrm{M}+\mathrm{Na}]^{+}$492.1246, found 492.1245 .

6-甲基-1-苯基-2-对甲苯磺酰基-5,6-二氢苯并 $[b]$ 吡 咯并 $[2,1-c][1,4]$ 惡嗪-3(7aH)-酮 $(3 \mathbf{j})$ : 白色固体, $28.6 \mathrm{mg}$, 产率 33\%. m.p. $191 \sim 192{ }^{\circ} \mathrm{C} ;{ }^{1} \mathrm{H}$ NMR $(400 \mathrm{MHz}$, $\left.\mathrm{CDCl}_{3}\right) \delta: 7.87(\mathrm{~d}, J=8.2 \mathrm{~Hz}, 2 \mathrm{H}), 7.42 \sim 7.36(\mathrm{~m}, 2 \mathrm{H})$, 7.30 (t, $J=8.3 \mathrm{~Hz}, 4 \mathrm{H}), 7.12(\mathrm{~d}, J=7.4 \mathrm{~Hz}, 2 \mathrm{H}), 6.23(\mathrm{dd}$, $J=9.4,5.4 \mathrm{~Hz}, 1 \mathrm{H}), 5.66 \sim 5.62(\mathrm{~m}, 1 \mathrm{H}), 5.56 \sim 5.52(\mathrm{~m}$, $1 \mathrm{H}), 5.27(\mathrm{~d}, J=9.9 \mathrm{~Hz}, 1 \mathrm{H}), 4.11(\mathrm{~s}, 1 \mathrm{H}), 3.95 \sim 3.91(\mathrm{~m}$, $1 \mathrm{H}), 3.26 \sim 3.21(\mathrm{~m}, 1 \mathrm{H}), 2.41(\mathrm{~s}, 3 \mathrm{H}), 1.16(\mathrm{~d}, J=6.6 \mathrm{~Hz}$, $3 \mathrm{H}) ;{ }^{13} \mathrm{C}$ NMR $\left(101 \mathrm{MHz}, \mathrm{CDCl}_{3}\right) \delta: 164.5,163.3,145.1$, $136.8,134.7,130.1,129.7,129.6,129.2,128.9,127.6$, 127.1, 126.3, 124.8, 122.5, 67.8, 66.4, 65.4, 42.9, 21.8, 15.6; IR (KBr) v: 3431, 3085, 3056, 2986, 2984, 2915, 1694, 1615, 1596, 1439, 1394, 1335, 1321, 1309, 1282, 1167, 1152, 1091, 1070, 1017, 985, 912, 815, 732, 693 $\mathrm{cm}^{-1}$; HRMS (ESI-TOF) calcd for $\mathrm{C}_{25} \mathrm{H}_{23} \mathrm{NO}_{4} \mathrm{NaS}[\mathrm{M}+$ $\mathrm{Na}]^{+}$456.1244, found 456.1245.

1-苯基-2-对甲苯磺酰基-6,7-二氢-苯并 $[b]$ 吡咯并

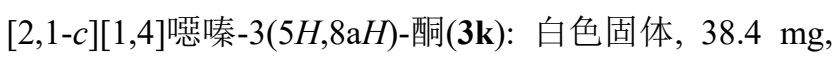
产率 44\%. m.p. $245 \sim 247{ }^{\circ} \mathrm{C}$; ${ }^{1} \mathrm{H}$ NMR $(400 \mathrm{MHz}$, $\left.\mathrm{CDCl}_{3}\right) \delta: 7.87(\mathrm{~d}, J=7.9 \mathrm{~Hz}, 2 \mathrm{H}), 7.38(\mathrm{~d}, J=7.2 \mathrm{~Hz}$, 1H), 7.31 (dd, $J=17.5,7.8 \mathrm{~Hz}, 4 \mathrm{H}), 7.17(\mathrm{~d}, J=7.5 \mathrm{~Hz}$, $2 \mathrm{H}), 6.29 \sim 6.25(\mathrm{~m}, 1 \mathrm{H}), 5.76 \sim 5.71(\mathrm{~m}, 1 \mathrm{H}), 5.58 \sim 5.55$ (m, 1H), $5.21(\mathrm{~d}, J=9.3 \mathrm{~Hz}, 1 \mathrm{H}), 4.19(\mathrm{~d}, J=4.0 \mathrm{~Hz}, 1 \mathrm{H})$, $4.10 \sim 4.05(\mathrm{~m}, 1 \mathrm{H}), 3.95 \sim 3.89(\mathrm{~m}, 1 \mathrm{H}), 3.61 \sim 3.55(\mathrm{~m}$, $1 \mathrm{H}), 3.06 \sim 3.00(\mathrm{~m}, 1 \mathrm{H}), 2.41(\mathrm{~s}, 3 \mathrm{H}), 2.07 \sim 2.00(\mathrm{~m}$, $1 \mathrm{H}), 1.76 \sim 1.70(\mathrm{~m}, 1 \mathrm{H}) ;{ }^{13} \mathrm{C} \mathrm{NMR}\left(101 \mathrm{MHz}, \mathrm{CDCl}_{3}\right) \delta$ : 165.0, 163.4, 1450.0, 136.9, 134.2, 129.9, 129.6, 129.5, 129.4, 129.0, 128.0, 127.5, 125.8, 124.7, 122.6, 75.9, 71.7, 68.5, 38.4, 28.3, 21.8; IR (KBr) v: 3442, 3054, 2933, 1693 , 
$1621,1594,1442,1397,1357,1332,1308,1187,1162$, 1146, 1099, 1073, 1029, 1010, 948, 813, 775, 772, 690, $663 \mathrm{~cm}^{-1}$; HRMS (ESI-TOF) calcd for $\mathrm{C}_{25} \mathrm{H}_{23} \mathrm{NO}_{4} \mathrm{NaS}$ $[\mathrm{M}+\mathrm{Na}]^{+}$456.1250, found 456.1245.

1-(对甲苯基)-2-对甲苯磺酰基-5,6-二氢苯并 $[b]$ 吡咯 并 $[2,1-c][1,4]$ 啞嗪-3(7a $H)$-酮(3I): 白色固体, $34.7 \mathrm{mg}$, 产率 40\%. m.p. $225 \sim 226{ }^{\circ} \mathrm{C}$; ${ }^{1} \mathrm{H}$ NMR $(400 \mathrm{MHz}$, $\left.\mathrm{CDCl}_{3}\right) \delta: 7.84(\mathrm{~d}, J=7.6 \mathrm{~Hz}, 2 \mathrm{H}), 7.28(\mathrm{~d}, J=7.5 \mathrm{~Hz}$, 2H), 7.08 (d, $J=7.1 \mathrm{~Hz}, 2 \mathrm{H}), 6.95$ (d, $J=7.2 \mathrm{~Hz}, 2 \mathrm{H}), 6.23$ $(\mathrm{d}, J=8.3 \mathrm{~Hz}, 1 \mathrm{H}), 5.57 \sim 5.61(\mathrm{~m}, 2 \mathrm{H}), 5.33 \sim 5.30(\mathrm{~m}$, $1 \mathrm{H}), 4.21 \sim 4.17(\mathrm{~m}, 1 \mathrm{H}), 3.84 \sim 3.82(\mathrm{~m}, 1 \mathrm{H}), 3.69(\mathrm{~s}$, $1 \mathrm{H}), 3.31 \sim 3.25(\mathrm{~m}, 1 \mathrm{H}), 3.11 \sim 3.05(\mathrm{~m}, 1 \mathrm{H}), 2.39(\mathrm{~s}$, 3H), $2.34(\mathrm{~s}, 3 \mathrm{H}) ;{ }^{13} \mathrm{C}$ NMR (101 MHz, $\left.\mathrm{CDCl}_{3}\right) \delta: 164.1$, $162.4,145.1,139.0,136.8,134.9,130.4,129.6,129.0$, $127.7,127.4,126.8,126.6,124.6,123.0,72.8,67.4,66.2$, 39.2, 21.8, 21.5; IR (KBr) v: 3377, 3052, 2966, 2918, 2854, 1697, 1621, 1596, 1505, 1435, 1387, 1331, 1307, 1289, 1193, 1162, 1087, 1062, 1047, 1019, 966, 892, 823, $814,768,746,703,685 \mathrm{~cm}^{-1}$; HRMS (ESI-TOF) calcd for $\mathrm{C}_{25} \mathrm{H}_{23} \mathrm{NO}_{4} \mathrm{NaS}[\mathrm{M}+\mathrm{Na}]^{+}$456.1245, found 456.1245.

1-(4-氯苯基)-2-对甲苯磺酰基-6,7-二氢-苯并 $[b]$ 吡 咯并[2,1-c][1,4]啞嗪-3(7aH)-酮(3m): 白色固体, $24 \mathrm{mg}$, 产率 26\%. m.p. $241 \sim 243{ }^{\circ} \mathrm{C}$; ${ }^{1} \mathrm{H}$ NMR $(400 \mathrm{MHz}$, $\left.\mathrm{CDCl}_{3}\right) \delta: 7.85(\mathrm{~d}, J=8.0 \mathrm{~Hz}, 2 \mathrm{H}), 7.32 \sim 7.26(\mathrm{~m}, 4 \mathrm{H})$, $7.05 \sim 7.01(\mathrm{~m}, 2 \mathrm{H}), 6.28 \sim 6.25(\mathrm{~m}, 1 \mathrm{H}), 5.67 \sim 5.63(\mathrm{~m}$, $2 \mathrm{H}), 5.34(\mathrm{~d}, J=9.4 \mathrm{~Hz}, 1 \mathrm{H}), 4.23 \sim 4.20(\mathrm{~m}, 1 \mathrm{H}), 3.87 \sim$ $3.69(\mathrm{~m}, 1 \mathrm{H}), 3.69(\mathrm{~s}, 1 \mathrm{H}), 3.33 \sim 3.28(\mathrm{~m}, 1 \mathrm{H}), 3.14 \sim$ $3.08(\mathrm{~m}, 1 \mathrm{H}), 2.42(\mathrm{~s}, 3 \mathrm{H}) ;{ }^{13} \mathrm{C} \mathrm{NMR}\left(101 \mathrm{MHz}, \mathrm{CDCl}_{3}\right) \delta$ : $162.2,162.0,145.4,136.5,135.8,135.4,130.6,129.7$, $129.0,129.0,128.3,127.4,126.8,124.7,122.98,72.77$, $67.28,66.26,39.22,21.75$; IR (KBr) v: 3380, 3084, 3053, 2971, 2867, 1698, 1617, 1597, 1488, 1387, 1330, 1308, 1288, 1185, 1161, 1088, 1072, 1061, 1015, 963, 844, 815, 767, 703, 680, 664, 649, $588 \mathrm{~cm}^{-1}$; HRMS (ESI-TOF) calcd for $\mathrm{C}_{24} \mathrm{H}_{20} \mathrm{NO}_{4} \mathrm{NaSCl}[\mathrm{M}+\mathrm{Na}]^{+} 520.0198$, found 520.0194 .

1-(4-溴苯基)-2-对甲苯磺酰基-6,7-二氢-苯并 $[b]$ 吡 咯并[2,1-c][1,4]惩嗪-3(7aH)-酮(3n): 白色固体, $21.9 \mathrm{mg}$, 产率 22\%. m.p. 226 228 ${ }^{\circ} \mathrm{C} ;{ }^{1} \mathrm{H}$ NMR $(400 \mathrm{MHz}$, $\left.\mathrm{CDCl}_{3}\right) \delta: 7.85(\mathrm{~d}, J=7.3 \mathrm{~Hz}, 2 \mathrm{H}), 7.44(\mathrm{~d}, J=7.4 \mathrm{~Hz}$, 2H), 7.31 (d, $J=7.4 \mathrm{~Hz}, 2 \mathrm{H}), 6.96$ (d, $J=7.1 \mathrm{~Hz}, 2 \mathrm{H}), 6.26$ $(\mathrm{d}, J=8.5 \mathrm{~Hz}, 1 \mathrm{H}), 5.73 \sim 5.62(\mathrm{~m}, 2 \mathrm{H}), 5.34(\mathrm{~d}, J=9.2$ $\mathrm{Hz}, 1 \mathrm{H}), 4.23 \sim 4.20(\mathrm{~m}, 1 \mathrm{H}), 3.87 \sim 3.84(\mathrm{~m}, 1 \mathrm{H}), 3.69(\mathrm{~s}$, $1 \mathrm{H}), 3.33 \sim 3.28(\mathrm{~m}, 1 \mathrm{H}), 3.14 \sim 3.07(\mathrm{~m}, 1 \mathrm{H}), 2.42(\mathrm{~s}$, $3 \mathrm{H}) ;{ }^{13} \mathrm{C}$ NMR (101 MHz, $\left.\mathrm{CDCl}_{3}\right) \delta: 162.1,162.0,145.4$,
$136.5,135.8,130.6,130.3,129.7,129.2,129.0,128.7$, $126.8,124.7,123.7,122.9,72.8,67.3,66.3,39.3,21.8$; IR (KBr) v: 3428, 3050, 2991, 2914, 2868, 1699, 1616, 1596, 1387, 1330, 1287, 1330, 1287, 1161, 1074, 1010, 962, 842, $814,705,663,640, \mathrm{~cm}^{-1}$; HRMS (ESI- TOF) calcd for $\mathrm{C}_{24} \mathrm{H}_{20} \mathrm{NO}_{4} \mathrm{NaSCl}[\mathrm{M}+\mathrm{Na}]^{+}$476.0701, found 476.0699.

4-(3-氧代-2-对甲苯磺酰基-3,5,6,7a-四氢苯并 $[b]$ 吡 咯并 $[2,1-c][1,4]$ 檼嗪-1-基)苯甲酸甲酯(3o): 白色固体， $19.1 \mathrm{mg}$, 产率 $20 \%$. m.p. 238 $239{ }^{\circ} \mathrm{C} ;{ }^{1} \mathrm{H}$ NMR $(400$ $\left.\mathrm{MHz}, \mathrm{CDCl}_{3}\right) \delta: 7.98(\mathrm{~d}, J=8.0 \mathrm{~Hz}, 2 \mathrm{H}), 7.85$ (d, $J=8.0$ $\mathrm{Hz}, 2 \mathrm{H}), 7.30$ (d, $J=7.9 \mathrm{~Hz}, 2 \mathrm{H}), 7.16(\mathrm{~d}, J=8.1 \mathrm{~Hz}, 2 \mathrm{H})$, $6.24(\mathrm{~d}, J=8.91 \mathrm{H}), 5.60 \sim 5.56(\mathrm{~m}, 2 \mathrm{H}), 5.36(\mathrm{~d}, J=9.3$ $\mathrm{Hz}, 1 \mathrm{H}), 4.23 \sim 4.19(\mathrm{~m}, 1 \mathrm{H}), 3.92(\mathrm{~s}, 3 \mathrm{H}), 3.87 \sim 3.84(\mathrm{~m}$, $1 \mathrm{H}), 3.71(\mathrm{~d}, J=4.1 \mathrm{~Hz}, 1 \mathrm{H}), 3.33 \sim 3.27(\mathrm{~m}, 1 \mathrm{H}), 3.15 \sim$ $3.08(\mathrm{~m}, 1 \mathrm{H}), 2.41(\mathrm{~s}, 3 \mathrm{H}) ;{ }^{13} \mathrm{C} \mathrm{NMR}\left(101 \mathrm{MHz}, \mathrm{CDCl}_{3}\right) \delta$ : $166.5,162.2,161.9,145.4,136.4,135.8,134.6,130.7$, $130.6,129.7,129.0,128.2,127.8,126.8,124.7,122.9$, 72.7, 67.3, 66.3, 52.4, 39.3, 21.8; IR (KBr) v: 3427, 2926, 2852, 1725, 1687, 1631, 1599, 1435, 1396, 1332, 1285, 1160, 1149, 1108, 1090, $588 \mathrm{~cm}^{-1}$; HRMS (ESI-TOF) calcd for $\mathrm{C}_{26} \mathrm{H}_{23} \mathrm{NO}_{6} \mathrm{NaSBr}[\mathrm{M}+\mathrm{Na}]^{+}$500.1147, found 500.1144 .

1-(噻吩-2-基)-2-对甲苯磺酰基-5,6-二氢苯并 $[b]$ 吡 咯并 $[2,1-c][1,4]$ 噁嗪-3(7aH)-酮 $(3 \mathbf{p})$ : 白色固体, $30.6 \mathrm{mg}$, 产率 36\%. m.p. $245 \sim 246{ }^{\circ} \mathrm{C}$; ${ }^{1} \mathrm{H}$ NMR $(400 \mathrm{MHz}$, $\left.\mathrm{CDCl}_{3}\right) \delta: 7.91(\mathrm{~d}, J=8.3 \mathrm{~Hz}, 2 \mathrm{H}), 7.45$ (dd, $J=4.0,2.2$ $\mathrm{Hz}, 1 \mathrm{H}), 7.31(\mathrm{~d}, J=8.4 \mathrm{~Hz}, 2 \mathrm{H}), 7.03 \sim 7.00(\mathrm{~m}, 2 \mathrm{H})$, $6.40 \sim 6.36(\mathrm{~m}, 1 \mathrm{H}), 5.78(\mathrm{dd}, J=9.6,5.4 \mathrm{~Hz}, 1 \mathrm{H}), 5.64$ $(\mathrm{dd}, J=9.6,5.5 \mathrm{~Hz}, 1 \mathrm{H}), 5.33 \sim 5.28(\mathrm{~m}, 1 \mathrm{H}), 4.22 \sim 4.18$ $(\mathrm{m}, 1 \mathrm{H}), 3.86 \sim 3.83(\mathrm{~m}, 1 \mathrm{H}), 3.70 \sim 3.68(\mathrm{~m}, 1 \mathrm{H}), 3.33 \sim$ $3.26(\mathrm{~m}, 1 \mathrm{H}), 3.13 \sim 3.06(\mathrm{~m}, 1 \mathrm{H}), 2.42(\mathrm{~s}, 3 \mathrm{H}) ;{ }^{13} \mathrm{C} \mathrm{NMR}$ $\left(101 \mathrm{MHz}, \mathrm{CDCl}_{3}\right) \delta: 162.0,156.8,145.3,136.5,136.4$, $131.3,130.2$, 129.6, 129.2, 128.2, 127.3, 126.5, 126.4, $124.3,122.2,73.1,66.9,66.0,39.2,21.8$; IR (KBr) v: 3447, 3119, 2966, 2852, 1702, 1612, 1594, 1387, 1327, 1308, 1160, 1085, 1069, 962, 917, 891, 824, 764, 742, 717, 688, 664, 649, $584 \mathrm{~cm}^{-1}$; HRMS (ESI-TOF) calcd for $\mathrm{C}_{22} \mathrm{H}_{19} \mathrm{NO}_{4} \mathrm{NaS}_{2}[\mathrm{M}+\mathrm{Na}]^{+}$448.0654, found 448.0653.

\section{3 荧光光谱测量方法}

配置含 $50 \mu \mathrm{mol} / \mathrm{L} \mathrm{3p}$ 的二氯甲烷溶液，并测试该溶 液的荧光发射光谱; 随后往溶液中加入 $\mathrm{Hg}^{2+}(50$ $\mu \mathrm{mol} / \mathrm{L}), 0.5 \mathrm{~h}$ 后测定其苂光光谱. 测试所用的激发波长 为 $365 \mathrm{~nm}$, 狭缝宽度为 $10 \mathrm{~nm} / 20 \mathrm{~nm}$.

辅助材料(Supporting Information) 化合物 31 的单晶 
数据、3a 3p 的核磁图谱. 这些材料可以免费从本刊网 站(http://sioc-journal.cn/)上下载.

\section{References}

[1] (a) Sietmann, J.; Wiest, J. M. Angew. Chem., Int. Ed. 2020, 59, 6964.

(b) Li, C.; Ragab, S.; Liu, G.; Tang, W. Nat. Prod. Rep. 2020, 37, 276.

(c) Pierrot, D.; Marek, I. Angew. Chem., Int. Ed. 2020, 59, 36.

(d) Yang, X.-W.; Grossman, R. B.; Xu, G. Chem. Rev. 2018, 118, 3508 .

(e) Wang, B.; Tu, Y.-Q. Acc. Chem. Res. 2011, 44, 1207.

[2] Nicolaou, K. C.; Baran, P. S.; Zhong, Y.-L.; Sugita, K. J. Am. Chem. Soc. 2002, 124, 2212.

[3] Parsons, A. F.; Williams, D. A. J. Tetrahedron 2000, 56, 7217.

[4] (a) Quiclet-Sire, B.; Zard, S. Z. Sci. Chin. Chem. 2019, 62, 1450.

(b) Zhu, Y.; Han, J.; Wang, J.; Shibata, N.; Sodeoka, M.; Soloshonok, V.; Coelho, J. A. S.; Toste, F. D. Chem. Rev. 2018, 118, 3887.

(c) Feng, J.; Holmes, M.; Krische, M. Chem. Rev. 2017, 117, 12564.

(d) Liu, Y.; Han, S.; Liu, W.-B.; Stoltz, B. Acc. Chem. Res. 2015, 48,740 .

[5] (a) Xia, Z.; Xu-Xu, Q.-F.; Zheng, C.; You, S. Chem. Soc. Rev. 2020, $49,286$.

(b) Zhang, Y.-C.; Jiang, F.; Shi, F. Acc. Chem. Res. 2020, 53, 425.

(c) Yan, Q.; Fan, R.; Liu, B.; Su, S.; Wang, B.; Yao, T.; Tan, J. Chin. J. Org. Chem. 2021, 41,455 (in Chinese).

(间强, 范荣, 刘斌斌, 苏帅松, 王勃, 姚团利, 谭嘉靖, 有机化 学, 2021, 41, 455.)

[6] (a) Liu, X.; Zheng, C.; Yang, Y.; Jin, S.; You, S. Angew. Chem., Int. Ed. 2019, 58, 10493.

(b) Chen, P.; Wu, Y.; Zhu, S.; Jiang, H.; Ma, Z. Org. Chem. Front. 2018, 5, 132.

(c) Zheng, C.; You, S. Chem 2016, 1, 830.

[7] Zhang, X.; Larock, R. C. J. Am. Chem. Soc. 2005, 127, 12230.

[8] (a) Husain, H.; Green, I. R.; Ahmed, I. Chem. Rev. 2013, 113, 3329.

(b) Zhang, M.-Z.; Liu, L.; Gou, Q.; Wang, Q.; Li, Y.; Li, W.-T.; He, W.-M. Green Chem. 2020, 22, 8369.

(c) Liu, X.; Li, L.; Jin, L.; Zhao, J.; Hua, Y.; Wang, M.; Liu, L. Chin. J. Org. Chem. 2020, 40, 4298 (in Chinese).

(刘新明, 李路, 晋李成, 赵金灿, 华远照, 王敏灿, 刘澜涛, 有 机化学, 2020, 40, 4298.)

[9] (a) Qiu, G.; Liu, T.; Ding, Q. Org. Chem. Front. 2016, 3, 510.

(b) He, Y.; Qiu, G. Z. Org. Biomol. Chem. 2017, 15, 3485.
[10] (a) Shi, L.; Zhang, W.; Chen, S.; Lu, L.; Fan, R.; Tan, J.; Zheng, C. Curr. Org. Synth. 2018, 15, 904.

(b) Vessally, E.; Babazadeh, M.; Didehban, K.; Hosseinian, A.; Edjlali, L. Curr. Org. Chem. 2018, 22, 286.

(c) Ni, S.; Zhou, J.; Mei, H.; Han, J. Tetrahedron Lett. 2018, 59, 1309.

(d) Reddy, C.; Prajapti, S.; Warudikar, K.; Ranjan, R.; Rao, B. Org. Biomol. Chem. 2017, 15, 3130.

(e) Song, R.; Xie, Y. Chin. J. Chem. 2017, 35, 280.

[11] (a) Wang, Y.-C.; Liu, J.-B.; Zhou, H.; Xie, W.; Rojsitthisak, P.; Qiu, G. J. Org. Chem. 2020, 85, 1906

(b) Wang, Y.-C.; Huang, K.-K.; Lai, X.-J.; Shi, Z.-N.; Liu J.-B.; Qiu, G. Org. Biomol. Chem. 2021, 19, 1940.

[12] (a) Simpkins, N. S. Oxford 1993.

(b) Noshi, M. N.; El-Awa, A.; Torres, E.; Fuchs, P. L. J. Am. Chem. Soc. 2007, 129, 11242.

(c) Kotha, S.; Chavan, A. S. J. Org. Chem. 2010, 75, 4319.

(d) Liu, K.-J.; Deng, J.-H.; Yang, J.; Gong, S.-F.; Lin, Y.-W.; He, J.-Y.; He, W.-M. Green Chem. 2020, 22, 433.

(e) Meng, X.-X.; Kang, Q.-Q.; Zhang, J.-Y.; Li, Q.; Wei, W.-T.; He, W.-M. Green Chem. 2020, 22, 1388.

(f) Liu, K.-J.; Wang, Z.; Lu, L.-H.; Chen, J.-Y.; Zeng, F.; Lin, Y.-W.; He, W.-M. Green Chem. 2021, 23, 496.

(g) Xie, L.-Y.; Fang, T.-G.; Tan, J.-X.; Zhang, B.; Cao, Z.; Yang, L.-H.; He, W.-M. Green Chem. 2019, 21, 3858.

(h) Qiu, G.; Zhou, K.; Gao, L.; Wu, J. Org. Chem. Front. 2018, 5, 691.

(i) Qiu, G.; Zhou, K.; Wu, J. Chem. Commun. 2018, 54, 12561.

(j) Qiu, G.; Lai, L.; Cheng, J.; Wu, J. Chem. Commun. 2018, 54, 10405.

[13] Wen, J.-W.; Wei, W.; Xue, S.-S.; Yang, D.-S.; Lou, Y.; Gao, C.-Y.; Wang, H. J. Org. Chem. 2015, 80, 4966.

[14] (a) Yuan, S.-T.; Zhou, H.; Gao, L.-L.; Liu, J.-B.; Qiu, G. Org. Lett. 2018, 20, 562 .

(b) Yuan, S.-T.; Zhou, H.-W.; Zhang, L.-P.; Liu, J.-B.; Qiu, G. Org. Biomol. Chem. 2017, 15, 4867.

(c) Wang, R.-X.; Yuan, S.-T.; Liu, J.-B.; Wu, J.; Qiu, G. Org. Biomol. Chem. 2018, 16, 4501.

(d) Wang, Y.-C.; Wang, R.-X.; Qiu, G.; Zhou, H.-W.; Xie, W.-L.; Liu, J.-B. Org. Chem. Front. 2019, 6, 2471.

(e) Wang, Y.-H.; Qiu, G.; Zhou, H.-W.; Xie, W.-L.; Liu, J.-B. Tetrahedron 2019, 75, 3850

(f) Wang, Y.-C.; Fang, Z.; Huang, K.-K.; Qiu, G.; Liu, J.-B. Mol. Catal. 2020, 495, 111163.

(g) Zhang, L.; Yuan, S.-T.; Wang, P.; Liu, J.-B. Chin. J. Org. Chem. 2020, 40, 1529 (in Chinese).

(张否，袁斯甜，王鹏，刘晋彪，有机化学，2020,40,1529.)

(Cheng, F.) 